\title{
A Green Protocol for the Synthesis of $\alpha$-Amino Phosphonates Catalyzed by Orange Peel Powder
}

\author{
Swati S. Ghodke ${ }^{1}$, Priya M. Khandare ${ }^{1}$, Ranjita D. Ingle ${ }^{1, *}$, Rajendra P. Pawar ${ }^{2, *}$ \\ Department of Chemistry, Deogiri College, Station Road, Aurangabad, Maharashtra - 431005, India \\ Department of Chemistry, Shiv Chhatrapati College, Aurangabad, Maharashtra - 431005, India \\ Correspondence: rppawar@yahoo.com (R.P.P.); rajitaingle@yahoo.in (R.D.I.); ghodkeswati0@gmail.com (S.S.G); \\ pmkhandare27@gmail.com (P.M.K.);
}

Scopus Author ID 7003738785

Received: 23.03.2021; Revised: 15.05.2021; Accepted: 19.05.2021; Published: 27.06.2021

Abstract: A simple and green protocol has been developed for the synthesis of $\alpha$-aminophosphonates from different substituted aromatic aldehydes, aniline and dimethyl phosphite in the presence of orange peel powder (OPP) as a natural catalyst under reflux conditions. The main advantages of this method are the high yield of product, simple workup procedure, short reaction time and easily available catalyst.

Keywords: Aldehyde; Aniline; Dimethyl Phosphite; Ethanol; Orange peel powder.

\section{Introduction}

$\alpha$-Aminophosphonates is an important class of organophosphorus compounds as their structures are similar to amino acids, where a carboxylic acid group is replaced by phosphoric acid [1]. $\alpha$-Aminophosphonic esters are the bioisosteres of $\alpha$-amino acids and have attracted attention due to a wide range of biological activities. They are important from industrial [2], biochemistry [3] and medicinal chemistry point of view [4].

$\alpha$-Aminophosphonates are biologically active compounds; and show various types of activities such as antibacterial [5], antiviral [6], anticancer [7], antibiotic [8], antitumor [9] and antiproliferative potential [10]. There are several methods for the synthesis of $\alpha$ aminophosphonates; among these, Kabachnik-Field's reaction constitutes the most convenient method [11].

Owing to the importance of $\alpha$-aminophosphonates, many scientists have developed various methods for the synthesis of $\alpha$-aminophosphonates by using different catalysts such as $\mathrm{HClO}_{4}-\mathrm{SiO}_{2}$ [12], $\mathrm{Zn}(\mathrm{OTf})_{2}$ [13], $\mathrm{PPA}-\mathrm{SiO}_{2}$ [14], cellulose- $\mathrm{SO}_{3} \mathrm{H}$ [15], $\mathrm{TaCl}_{5}-\mathrm{SiO}_{2}$ [16], succinic acid [17], $\mathrm{H}_{3} \mathrm{PW}_{12} \mathrm{O}_{40}$ [18], oxalic acid [19] and aluminium nitride [20].

In the present work, we report an eco-friendly and clean route for one-pot synthesis of $\alpha$-aminophosphonates by using them as a simple natural catalyst. Here we used orange peel powder (OPP), which is a natural, efficient and heterogeneous catalyst. Orange is a popular source of vitamin $\mathrm{C}$ [21]. The orange peel powder mainly consists of soluble sugars $(16.9 \%$ wt.\%), starch (3.75\% wt.\%), ashes (3.50\% wt.\%), fats (1.95\% wt.\%) and protein (6.50\% wt\%). [22]. In continuation of our research work, we wish to report an eco-friendly synthetic route for the one-pot synthesis of $\alpha$-aminophosphonates by reacting aromatic aldehyde, aniline, and dimethyl phosphite in the presence of a catalytic amount of OPP in ethanol solvent under reflux condition in a short reaction time. 


\section{Materials and Methods}

All the melting points were determined in open capillaries and were uncorrected. The products were confirmed by comparing their physical data with literature values. ${ }^{1} \mathrm{H}$ NMR, and MASS spectra. ${ }^{1} \mathrm{H}$ NMR spectra were recorded on a BRUKER AVANCE NEO 500MHz NMR spectrometer. Chemical shifts were reported in $\delta \mathrm{ppm}$ by using tetramethylsilane as the internal standard in $\mathrm{CDCl}_{3}$ solvent. The progress of the reaction was monitored by TLC (n-hexane: ethyl acetate, 7:3).

\subsection{Synthesis of $\alpha$-aminophosphanates}

A mixture of aromatic aldehyde $(1 \mathrm{mmol})$ and aniline $(1 \mathrm{mmol})$ and dimethyl phosphite $(1.2 \mathrm{mmol})$ in ethanol solvent and orange peel powder (OPP) (10 wt\%) as a natural catalyst was added and reflux for the appropriate time. The progress of the reaction was monitored by TLC (n-hexane:ethyl acetate, 7:3). After completion of the reaction, the solvent of ethanol from the reaction mixture was evaporated and the reaction mixture was poured onto ice-cold water and filtered off. The resulting solid was further purified by recrystallization from ethanol (Scheme 1).

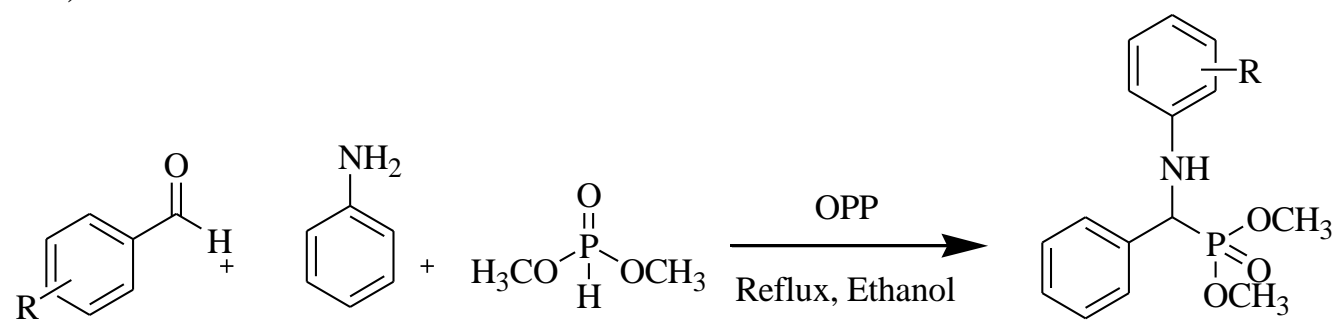

Scheme 1. Synthesis of $\alpha$-aminophosphonates.

Using the above protocol, several aldehydes were treated with aniline to afford the corresponding $\alpha$-aminophosphanates (Table 1).

Table 1. Synthesis of $\alpha$-aminophosphonates derivatives.

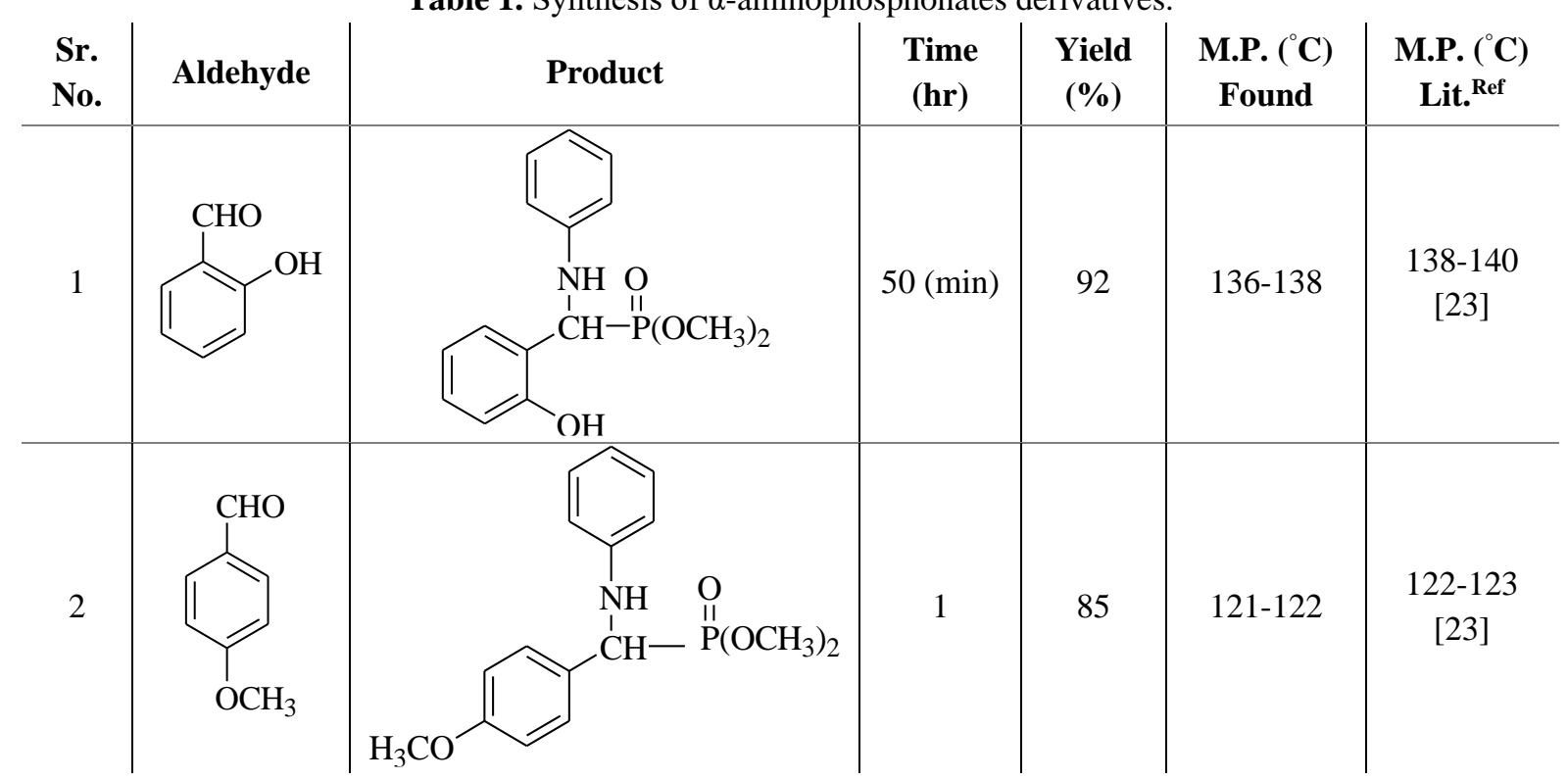




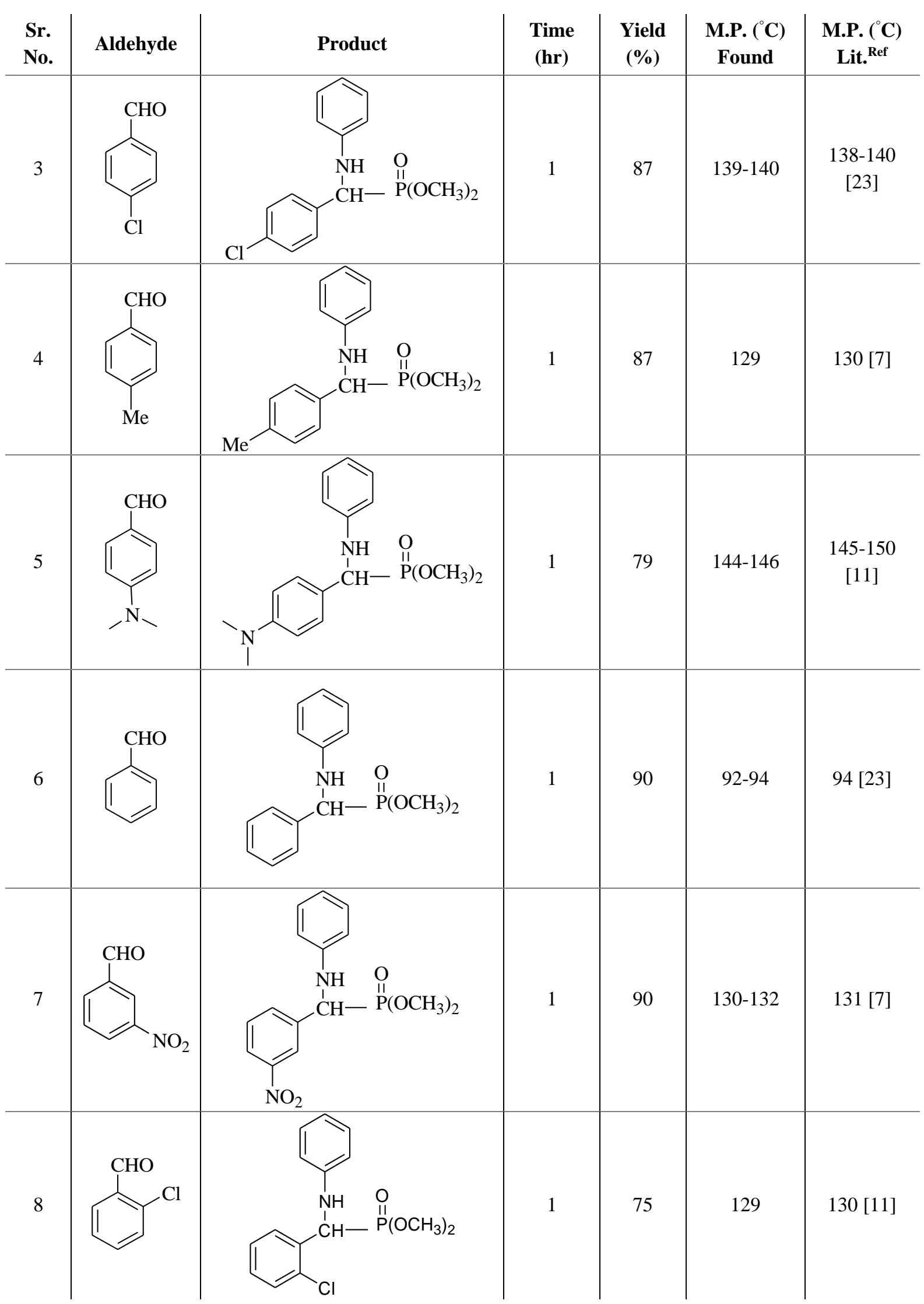

Compound 1. (Entry 1) ${ }^{1} \mathrm{H}$ NMR (500 MHz, $\left.\mathrm{CDCl}_{3}\right) \delta \mathrm{ppm} 7.02(\mathrm{dd}, 2 \mathrm{H}), 6.89(\mathrm{dd}, 2 \mathrm{H}), 6.86$ $(\mathrm{d}, 1 \mathrm{H}), 6.72(\mathrm{dd}, 1 \mathrm{H}), 6.60(\mathrm{~d}, 1 \mathrm{H}), 6.52(\mathrm{~m}, 1 \mathrm{H}), 6.40(\mathrm{~d}, 2 \mathrm{H}), 5.03(\mathrm{~d}, 1 \mathrm{H}), 4.2(\mathrm{br}, \mathrm{s}, 1 \mathrm{H})$, 4.0(s, 1H), 3.37(s, 1H), GC-MS: $308.20(\mathrm{M}+1)^{+}$.

Compound 2. (Entry 2) ${ }^{1} \mathrm{H}$ NMR (500 MHz, CDCl 3$) \delta$ ppm 6.50-7.34 (m, 9H), $4.75(\mathrm{~d}, 1 \mathrm{H})$, 4.70(br, s, 1H), 3.75 (s, 3H), $3.73(\mathrm{~s}, 3 \mathrm{H}), 3.45(\mathrm{~d}, 3 \mathrm{H})$, GCMS: $337.12(\mathrm{M}+1)^{+}$ 
Compound 3. (Entry 3) ${ }^{1} \mathrm{H}$ NMR (500 MHz, CDCl 3 ) $\delta$ ppm 6.45-7.34 (m, 1H, Ar-H), 4.71 (br, s, 1H), $4.69(\mathrm{~d}, 3 \mathrm{H}), 3.42(\mathrm{~d}, 3 \mathrm{H})$, GCMS: $342.08(\mathrm{M}+1)^{+}$

Compound 4. (Entry 4) ${ }^{1} \mathrm{H}$ NMR $\left(500 \mathrm{MHz}, \mathrm{CDCl}_{3}\right) \delta \mathrm{ppm} 7.40(\mathrm{~d}, 2 \mathrm{H}), 7.08-7.17(\mathrm{~m}, 4 \mathrm{H})$, 6.60-6.74 (m, 3H), 4.84 (br, S, 1H,), 4.79 (d, 1H), 3.74 (d, 3H), 3.50 (d, 3H), $2.30(\mathrm{~S}, 3 \mathrm{H})$, GCMS: $321.12(\mathrm{M}+1)^{+}$

Compound 5. (Entry 5) ${ }^{1} \mathrm{H}$ NMR (500 MHz, $\left.\mathrm{CDCl}_{3}\right) \delta \mathrm{ppm} 7.30(\mathrm{t}, 2 \mathrm{H}), 7.10(\mathrm{~m}, 2 \mathrm{H}), 6.65$ $(\mathrm{m}, 3 \mathrm{H}), 6.60(\mathrm{~d}, \mathrm{~d}, 2 \mathrm{H}), 4.71(\mathrm{~d}, 2 \mathrm{H}), 3.75(\mathrm{~d}, 3 \mathrm{H}), 2.91(\mathrm{~s}, 6 \mathrm{H}), 2.13(\mathrm{~s}, 1 \mathrm{H})$, GCMS: 350.17 $(\mathrm{M}+1)^{+}$

Compound 6. (Entry 6) ${ }^{1} \mathrm{H}$ NMR (500 MHz, $\left.\mathrm{CDCl}_{3}\right) \delta \mathrm{ppm}$ 7.25-8.01(m, 7H), 5.91(d, $\left.1 \mathrm{H}\right)$, $5.80(\mathrm{~m}, 1 \mathrm{H}), 3.90(\mathrm{~d}, 3 \mathrm{H}), 3.45(\mathrm{~d}, 3 \mathrm{H}), \mathrm{GCMS}: 307.10(\mathrm{M}+1)^{+}$

Compound 7. (Entry 7) ${ }^{1} \mathrm{H} \mathrm{NMR}\left(500 \mathrm{MHz}, \mathrm{CDCl}_{3}\right) \delta \mathrm{ppm}{ }^{1} \mathrm{H} \mathrm{NMR}\left(500 \mathrm{MHz}, \mathrm{CDCl}_{3}\right) \delta$ ppm $8.40(\mathrm{~d}, 1 \mathrm{H}), 8.20(\mathrm{~d}, 1 \mathrm{H}), 7.90(\mathrm{~d}, 1 \mathrm{H}), 7.59(\mathrm{t}, 1 \mathrm{H}), 7.25(\mathrm{t}, 2 \mathrm{H}), 6.75(\mathrm{t}, 1 \mathrm{H}), 6.63(\mathrm{~d}$, 2H) 5.32 (br, s, 1H), $4.93(\mathrm{~d}, 1 \mathrm{H}), 3.80(\mathrm{~d}, 3 \mathrm{H}), 3.60(\mathrm{~d}, 3 \mathrm{H})$, GCMS: $352.12(\mathrm{M}+1)^{+}$

Compound 8. (Entry 8) ${ }^{1} \mathrm{H}$ NMR (500 MHz, $\left.\mathrm{CDCl}_{3}\right) \delta$ ppm $6.60(\mathrm{~d}, \mathrm{~m}, 9 \mathrm{H}), 5.34(\mathrm{~d}, 1 \mathrm{H}), 4.90$ (br, 1H), $3.61(\mathrm{~d}, 3 \mathrm{H}), 3.41(\mathrm{~d}, 3 \mathrm{H})$, GCMS: $341.10(\mathrm{M}+1)^{+}$

\section{Results and Discussion}

A model condensation reaction of 2-hydroxy benzaldehyde ( $1 \mathrm{mmol})$, aniline ( $1 \mathrm{mmol})$, and dimethyl phosphite $(1.2 \mathrm{mmol})$ were selected for optimization of the reaction conditions (Scheme 2). Initially, the solvent effect on the model reaction was studied under reflux conditions. A trace amount of the product was obtained in the model reaction under the solventfree condition at reflux condition. Other solvents like water, ethanol, and toluene were also tried for the reaction (Table 2). Among the solvents, ethanol was the best solvent for the model reaction and afforded the product with excellent yields.<smiles>CCOc1cccc(C(Nc2ccccc2)P(=O)(OC)OC)c1O</smiles>

Scheme 2. General reaction for the synthesis of $\alpha$-aminophosphonates derivatives.

Table 2. Optimization of reaction solvent condition for the synthesis of $\alpha$-aminophosphonates derivatives.

\begin{tabular}{c|c|c|c} 
Solvent & Time $(\mathbf{h r})$ & Amount of catalyst $(\mathbf{w t} \%)$ & Yield $(\boldsymbol{\%})$ \\
\hline No solvent & 2.5 & 10 & Trace \\
\hline Water & 2 & 10 & 60 \\
\hline Ethanol & $50(\mathrm{~min})$ & 10 & 92 \\
\hline Toluene & 2 & 10 & 58
\end{tabular}

Next, we investigated the catalyst optimization on the model reaction of 2 hydroxybenzaldehyde, aniline and dimethyl phosphite in the presence of orange peel powder in ethanol solvent. The amount of catalyst used was varied from 5, 10, 15, $20 \mathrm{wt} \%$. Results showed that $10 \mathrm{wt} \%$ of the catalyst was sufficient enough to carry this reaction. We obtained an excellent yield of $92 \%$ for $10 \mathrm{wt} \%$ of the catalyst in $50 \mathrm{~min}$. Further increase in the catalyst concentration did not affect the yield (Table 3). 
Table 3. Optimization of catalyst concentration on the synthesis of $\alpha$-aminophosphonates.

\begin{tabular}{c|c|c} 
Entry & Catalyst concentration $(\mathbf{w t} \%)$ & Yield $(\boldsymbol{\%})$ \\
\hline 1 & 5 & 40 \\
\hline 2 & 10 & 92 \\
\hline 3 & 15 & 93 \\
\hline 4 & 20 & 93
\end{tabular}

\section{Conclusion}

In the present work, we report an efficient, green, and eco-friendly synthesis of $\alpha$ aminophosphonates by using different substituted aromatic aldehydes, aniline and dimethyl phosphite in ethanol solvent using orange peel powder at reflux condition. OPP is a natural and heterogeneous catalyst. The advantages of this method are excellent yield, short reaction time and simple procedure.

\section{Funding}

This paper has no external funding.

\section{Acknowledgments}

Our sincere thanks to the Principal, Deogiri College, Aurangabad, for providing necessary laboratory facilities.

\section{Conflict of interest}

The authors declare no conflict of interest.

\section{References}

1. Basha, M.H.; Chennamasetty, S.; Rao, K.P. Ultrasound promoted solvent-free synthesis of some new $\alpha-$ aminophosphonates as potential antioxidant, Main Group Metal Chem 2020, 43, 147-153, https://doi.org/10.1515/mgmc-2020-0018

2. Rezaei, Z.; Khabnadideh, S.; Zomorodian, K.; Pakshir, K.; Nadali, S.; Mohtashami N.; Mirzaei E.F. Design, synthesis and antifungal activity of new $\alpha$-aminophosphonates, International Journal of Medicinal Chemistry, 2011, 2011, 11, https://doi.org/10.1155/2011/678101

3. Balint, E.; Tripolszky, A.; Tajti, A. 6. Synthesis of $\alpha$-aminophosphonates by the Kabachnik-fields reaction and by the Pudovik reaction, in Organophosphorus Chemistry; Keglevich, G., Ed.; Berlin, Boston: De Gruyter, 2018, 108-147, https://doi.org/10.1515/9783110535839-006

4. Dake, S.A.; Raut, D.S.; Kharat, K.R.; Mhaske, R.S.; Deshmukh, S.U.; Pawar, R.P. Ionic liquid promoted synthesis, antibacterial and in vitro antiproliferative activity of novel $\alpha$-aminophosphonates derivatives, Bioorganic and Medicinal Chemistry Letters 2011, 21, 2527-2532, https://doi.org/10.1016/j.bmcl.2011.02.039

5. Carraminana, V.; Ochoa de Retana, A.M.; Palacios, F.; de los Santos J.M. Synthesis of $\alpha$ - aminophosphonic acid derivatives through the addition of $\mathrm{O}$-and $\mathrm{S}$-nucleophiles to $2 \mathrm{H}$-azirines and their antiproliferative effect on A549 human lung adenocarcinoma cells, Molecules 2020, 25, 3332, https://doi.org/10.3390/molecules25153332

6. Mandha, S.R.; Alla, M.; Bommena V.R. Water mediated eco-friendly green protocol for one pot synthesis of $\alpha$-aminophosphonates at ambient condition, $J$ Chem Sci 2014, 126, 793-799, https://doi.org/10.1007/s12039-014-0608-x

7. Movassag, B.; Alapour, S. P- dodecylbenznesulfonic acid: A highly efficient catalyst for one pot synthesis of $\alpha$-Aminophosphonates in aqueous media, Heteroatom chemistry 2013, 24, 174-178, https://doi.org/10.1002/hc.21079

8. Nagargoje, D.R.; Mandhane, P.G.; Bhagat, S.B.; Chavan, N.M.; Badadhe, P.V.; Gill, C. H. Synthesis of and 
characterization of $\alpha$-aminophosphonates of fluorinated pyrazole imines using triethyl phosphite and TMSCl, Der pharma Chemica, 2011, 3, 1-6

9. Shastri, R.A. Review on the synthesis of $\alpha$-aminophosphonate derivatives, Chem Sci Trans 2019, 8, 359367, https://doi.org/10.7598/cst2019.1585

10. Deshmukh, S.U.; Kharat, K.R.; Yadav, A.R.; Shisodia, S.U.; Damale, M.G.; Sangshetti, J.N.; Pawar, R.P. Synthesis of novel $\alpha$-aminophosphonate derivatives, biological evaluation as potent antiproliferative agent and molecular docking, ChemistrySelect 2018, 3, 5552-5558, https://doi.org/10.1002/slct.201800798

11. Mohammadiyan, E.; Ghafuri, H.; Kakanejadifard, A. A new procedure for synthesis of $\alpha$-aminophosphonate by aqueous formic acid as an effective and environment-friendly organocatalyst, J Chem Sci 2017, 129, 1883-1891, https://doi.org/10.1007/s12039-017-1394-z

12. Maghsoodlou, M.T.; Habibi-Khorassani, S.M.; Heydari, R.; Hazeri, N.; Sajadikhah, S.S.; Rostamizadeh, M. An efficient and simple synthesis of $\alpha$-aminophosphonate as 'drug like' molecule catalyzed by silica supported perchloric acid $\left(\mathrm{HClO}_{4}-\mathrm{SiO}_{2}\right)$, Arabian Journal of Chemistry 2011, 4, pp. 481-485, https://doi.org/10.1016/j.arabjc.2010.07.010

13. Essid, I.; Touil, S. Efficient and green one-pot multi-component synthesis of $\alpha$-aminophosphonates catalyzed by Zinc triflate, Current organic synthesis 2017, 14, 272-278, https://doi.org/10.2174/1570179413666160624092814

14. Maghsoodlou, M.T.; Habibi Khorassani, S.M.; Heydari, R.; Hazeri, N.; Sajadikhah, S.S.; Rostamizadeh, M.; Keishams, L. Silica supported polyphosphoric acid (PPA-SiO2): an efficient and reusable heterogeneous catalyst for the one pot synthesis of $\alpha$-aminophosphonates, Turk $J$ Chem 2010, 34, 565-570, https://doi.org/10.3906/kim-0910-28

15. Kumar, K.S.; Krishna, B.S.; Reddy, C.B.; Reddy, M.V.N.; Reddy, C.S. Solvent-free synthesis of $\alpha$ aminophosphonates: Cellulose- $\mathrm{SO}_{3} \mathrm{H}$ as an efficient catalyst, Arabian Journal of Chemistry 2012, 10, S368S375, https://doi.org/10.1016/j.arabjc.2012.09.009

16. Chandrasekhar, S.; Jaya Prakash, S.; Jagadeshwar, V.; Narsihmulu, Ch., Three component coupling catalyzed by TaCl5-SiO2: synthesis of $\alpha$-amino phosphonates, Tetrahedron Letters 2001, 42, 5561-5563, https://doi.org/10.1016/S0040-4039(01)01053-X

17. Hazeri, N.; Maghsoodlou, M.T.; Habibi-Khorassani, S.M.; Aboonajmi, J.; Lashkari, M.; Sajadikhah, S.S. A green protocol for one pot 3-compount synthesis of $\alpha$-aminophosphonates catalyzed by succinic acid, Research on Chemical Intermediates 2014, 40, 1781-1788, https://doi.org/10.1007/s11164-013-1081-8

18. Heydari, A.; Hamadi, H.; Pourayoubi, M. A new one-pot synthesis of $\alpha$-aminophosphonates catalyzed by $\mathrm{H}_{3} \mathrm{PW}_{12} \mathrm{O}_{40}$, Catalysis Communications 2007, 8, 1224-1226, https://doi.org/10.1016/j.catcom.2006.11.008

19. Vahdat, S.M.; Baharfar, R.; Tajbakhsh, M.; Heydari, A.; Baghbanian, S.M.; Khaksar, S. Organocatalytic synthesis of $\alpha$-hydroxy and $\alpha$-aminophosphonates, Tetrahedron Letters 2008, 49, 6501-6504, https://doi.org/10.1016/j.tetlet.2008.08.094

20. Tekale, S.U.; Kauthale, S.S.; Shaikh, R.U.; Marathe, R.P.; Nawale, T.B.; Pawar, R.P. Aluminiun nitride catalyzed solvent-free synthesis of some novel biologically active $\alpha$-aminophosphonates, Journal of the Iranian Chemical Society 2014, 11, 717-724, https://doi.org/10.1007/s13738-013-0344-z

21. Raj, A.; Masih, D. Physico chemical and rheological properties of wheat flour bun supplemented with orange peel powder, IJSR 2014, 3, 391-394

22. Torrado, A.M.; Cortes, S.; Salgado, J.M.; Max, B.; Rodriguez, N.; Bibbins, B.P.; Converti, A.; Dominguez, J.M. Citric acid production from orange peel wastes by solid-state fermentation, Braz J Microbiol 2011, 42, 394-409, https://doi.org/10.1590/S1517-83822011000100049

23. Khandare, P.M.; Ghodke, S.S.; Dixit, R.R.; Ingle, R.D.; Pawar, R.P. Synthesis of $\alpha$-aminophosphonate using lemon peel powder as an efficient catalyst, JETIR 2020, 7, 30-34 